\title{
Inelastic neutron scattering with GAINS at GELINA: An overview of the last decade
}

\author{
Catalin Borcea $^{1, a}$, Philippe Dessagne ${ }^{2}$, Maëlle Kerveno ${ }^{2}$, Cristian Mihailescu $^{1,3}$, Nikolay Nankov ${ }^{3}$, Alexandru Negret $^{1}$, \\ Markus Nyman ${ }^{3}$, Adina Olacel $^{1}$, Laszlo Oláh $^{3}$, Arjan Plompen $^{3}$, Chariklia Rouki $^{3}$, and Gerard Rudolf ${ }^{2}$ \\ ${ }^{1}$ Horia Hulubei National Institute for Physics and Nuclear Engineering, Bucharest-Magurele, Romania \\ 2 Unistra, CNRS, IPHC, Strasbourg, France \\ ${ }^{3}$ European Commission, Joint Research Centre, Geel, Belgium
}

\begin{abstract}
GAINS is an array of HPGe detectors installed at the GELINA neutron source of Joint Research Center, Geel, Belgium. It served to measure the cross section for (n,n' $\gamma$ ) reactions on many isotopes. The paper presents the experimental setup and reviews the main results obtained in the last decade
\end{abstract}

\section{Introduction}

The inelastic scattering is the main mechanism by which neutrons lose energy in a fission reactor. Therefore, knowing the cross sections for these process is essential for determining the economy of neutrons with different energies inside a reactor. Besides, inelastic scattering generates $\gamma$ rays that enter in the thermal balance of a reactor and are also important for radiation safety. Today, the GEN IV reactors [1] are designed on the basis of simulation codes (e.g. MCNP) and their related input data. Both components are important and continuously improved. Changing deliberately the input data by a certain amount and analyzing the consequence of this change on the output responses one can determine the acceptable limits for the errors in the data. High standards are therefore imposed on the quality of the data and inelastic neuron cross sections are an important part of it. A priority list of isotopes has been elaborated (High Priority Request List [2]) and this list guided our measurements. Besides, our results comply with the standards for quality: the evaluated uncertainties for each specific case, are generally kept below 5\%, and the sensitivity for the $\gamma$-production cross sections (which is the primarily measured quantity) is around $10 \mathrm{mb}$ for which we have a high resolution for the incident neutron energy.

\section{Experiment}

In order to perform the neutron inelastic cross section experiments we measure the $\gamma$-production cross sections for the most important transitions from the nucleus populated following the inelastic scattering of neutrons. Using the known decay scheme of the studied isotope from the ENSDF data base [3], these cross sections serve to obtain, firstly the level population cross sections and then

\footnotetext{
a e-mail: borcea@nipne.ro
}

finally the total inelastic cross sections. The experiment requires:

- a good quality neutron beam (intense, pulsed with a short pulse duration) followed by a flight path for time of flight (TOF) measurements;

- a precise and reliable monitoring system for the neutron beam;

- an adequate detection and data acquisition system (DAQ).

\subsection{The neutron beam}

The neutron beam is produced by GELINA (Geel Electron Linear Accelerator) [4-6]. An accelerated (LINAC) electron beam is firstly time-compressed using a $180^{\circ}$ magnet, such as to make the pulse as short as possible (typically $1 \mathrm{~ns}$ ) in order to achieve the highest resolution for the incident energy by time of flight. Then it is sent on a $U$ target. This is a cooled rotating disk that stops the beam about $3 \mathrm{~cm}$ after impact. The neutrons are abundantly produced by $(\gamma, \mathrm{n})$ and $(\gamma, \mathrm{f})$ reactions and fly through a number of evacuated flight paths with various lengths (from 10 to $400 \mathrm{~m}$ ). Our experiment is located on the flight path 3 (FP3), initially at $200 \mathrm{~m}$ and recently moved to $100 \mathrm{~m}$ from the neutron production target. GELINA can be operated at different repetition rates for the pulses; most of our measurements were done at the highest repetition rate of 800 pulses/s. In our TOF measurements, the time resolution is directly reflected into the resolution of the measured neuron energy. The width of the GELINA pulses (1 ns) is much smaller than the time resolution of our HPGe detectors (10 ns) which determines a variable energy resolution for neutrons: from $1.4 \mathrm{keV}$ for $1 \mathrm{MeV}$ neutrons to $44 \mathrm{keV}$ for $10 \mathrm{MeV}$ neutrons (200$\mathrm{m}$ flight path). This energy resolution is sufficient not only to follow the structures appearing in the shape of the excitation functions at low energy but also to get an idea about level densities for some light elements (resonant 


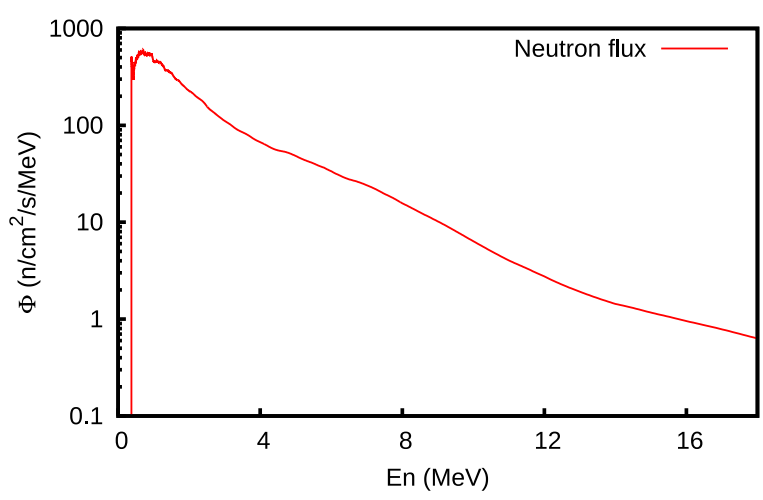

Figure 1. The neutron flux in the cabin located on FP3 at $200 \mathrm{~m}$ from the neutron producing target [7].

structures can be counted individually). Along the flight path two filters are placed at mid distance to the target: a 2-cm thick $U$ filter for diminishing the intensity of the $\gamma$ flash issued at the impact of electrons on the GELINA production target and a ${ }^{10} \mathrm{Be}$ filter for eliminating the slow neutrons that may overlap a previous pulse at the target location. Before the target, the neutron flux is defined by a long collimator to a parallel, homogeneous beam with a diameter of $61 \mathrm{~mm}$. Figure 1 shows the shape of the neutron spectrum obtained after collimation in the cabin at $200 \mathrm{~m}$ on FP3. For neutron energies below $4 \mathrm{MeV}$ (region that covers the first excited state in most nuclei) the neutron flux is the highest, allowing the observation of individual structures. The exponential fall in intensity limits our measurements to energies around $15 \mathrm{MeV}$. In that region the inelastic cross section has rather smooth behaviour allowing us to group several neutron energy bins and therefore to improve statistics.

The monitoring of the beam is made by a fission chamber (FC) placed $148.8 \mathrm{~cm}$ upstream the target. The FC has 5 circular Al plates that bear 8 deposits of ${ }^{235} \mathrm{U}$. The FC is operated with a continuous flow of P10 gas (argon and methane mixture) at the atmospheric pressure. The $\alpha$ activity events of ${ }^{235} \mathrm{U}$ overlap the recorded fission events induced by neutrons in the region of small amplitudes and therefore one should be careful to make a correct separation of the two contributions. A thorough study [8] resulted in a recipe to extrapolate toward very low energies. This procedure brings a $1 \%$ contribution to the systematic uncertainty of our measurements.

\subsection{Targets and detection}

The targets ranged from ${ }^{7} \mathrm{Li}$ to ${ }^{209} \mathrm{Bi}$ (see Table 1). Some of them needed a special treatment, e.g. Na targets that were shaped and then encapsulated in $\mathrm{Al}$ under Ar atmosphere. We also used chemical compounds like $\mathrm{SiO}_{2}$ and $\mathrm{LiF}_{4}$, and in some cases natural isotopes, for which the thicknesses varied from 1 to $6 \mathrm{~mm}$. Object of continuous improvements, the detectors are placed in the rear hemisphere to minimize the $\gamma$-flash effects. The angles, $110^{\circ}$ and $150^{\circ}$ are nodes of the $4^{\text {th }}$ degree Legendre polynomials allowing us to perform the angular integration of the $\gamma$-production cross section. Later we added also detectors at $125^{\circ}$. Today we use 12 HPGe detectors of $100 \%$ relative efficiency supported by a special structure: Gamma Array for Inelastic Neutron Scattering, GAINS (see Fig. 2) [9,10]. Presently the detectors are installed

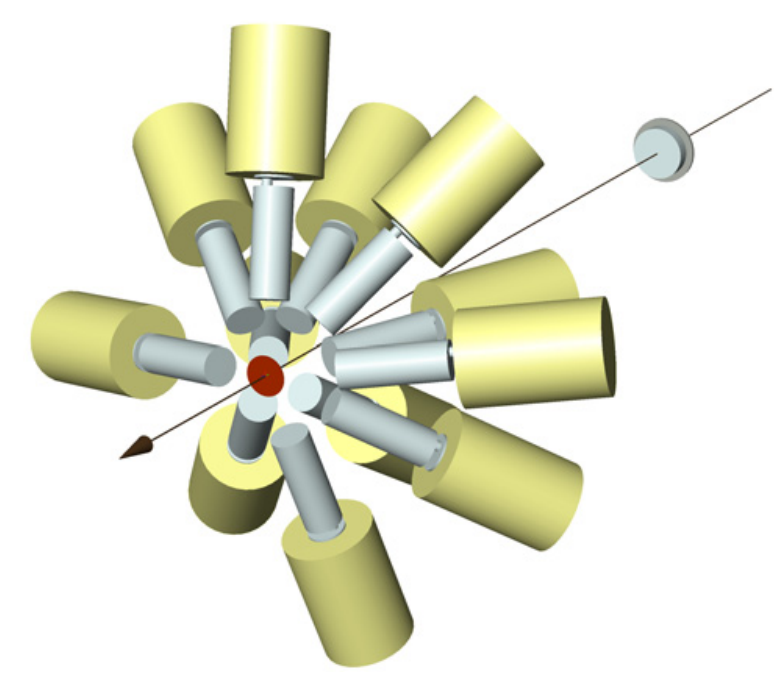

Figure 2. The schematically drawing of the GAINS setup installed in the measurement cabin located on FP3 at $100 \mathrm{~m}$ from the neutron production target.

in the cabin at $100 \mathrm{~m}$. The distances from the center of the sample to the front of detectors were around $14 \mathrm{~cm}$ at $200 \mathrm{~m}$ and $17 \mathrm{~cm}$ at $100 \mathrm{~m}$.

\subsection{Data acquisition and treatment}

The DAQ system evolved from analogical to digital. Presently we use Acqiris DC440 (420 Mb/s, 12 bits) digitizers, one channel for each detector. An analysis algorithm has been developed that produces an optimal time and energy resolution: $10 \mathrm{~ns}$ and $2.8 \mathrm{keV}$ (at $\mathrm{E}_{\gamma}=1332 \mathrm{keV}$ ). The typical load during the experiments is $10-20$ events/s, therefore we can afford to transfer the digitized signals to the main computer that can process them and write on disk only the useful information for each event: arrival time and amplitude. The Ge detectors are calibrated in energy and efficiency following a rigorous procedure: a modeling with MCNP and/or GEANT codes is compared to the measurements using calibrated point sources and assuming the geometry parameters, such as the distances to the sample, are finely tuned. Subsequently, a simulated efficiency for an extended source (sample) is produced to be used for data analysis. In some cases the procedure has been validated also with sets of specially prepared calibrated, extended sources. The systematic errors produced by these procedures are an important part in the total uncertainty and it was estimated at 2-3\%. Another correction applied to the data, especially important for thick targets, refers to the neutron multiple scattering. For this corrections the MCNP code was used. A detailed description of the procedures used for data reduction and for obtaining the cross sections is given in Ref. [11].

\section{Results}

The performed measurements and corresponding references are indicated in Table 1. In the last column some observations are given. Here only some particularities will be highlighted as well as some interesting, collateral aspects.

In all cases our results were compared with the calculations performed with the TALYS code [24] and 


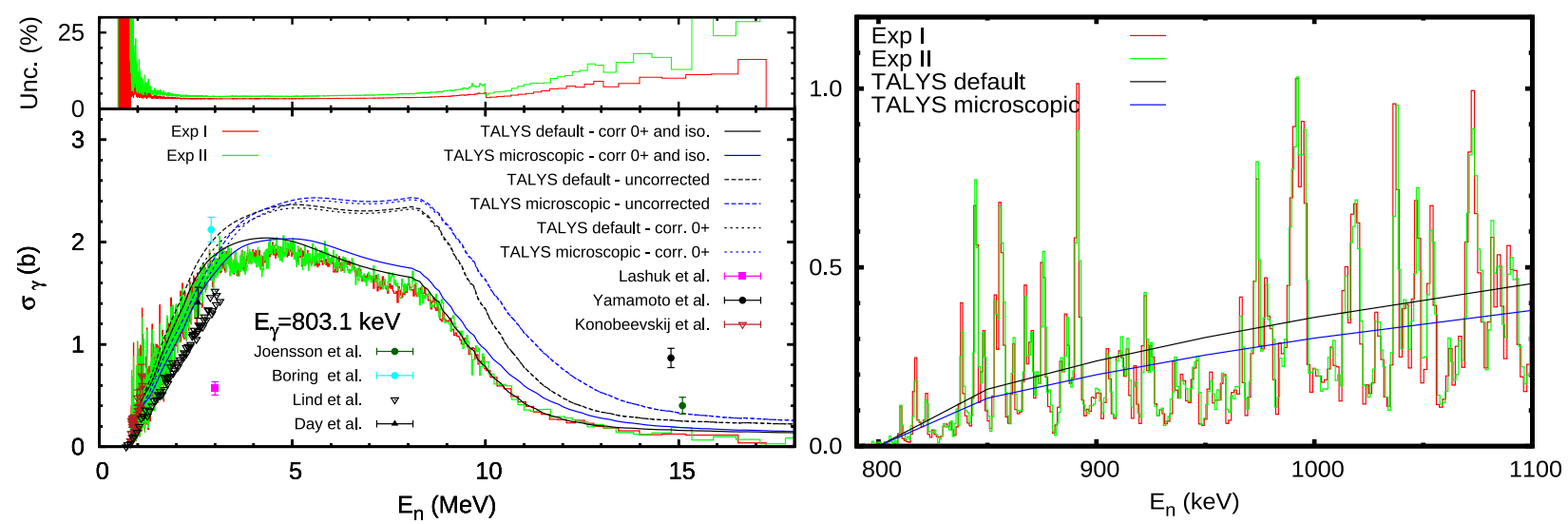

Figure 3. Top left: the corresponding total relative uncertainties of the (bottom left) integral $\gamma$-production cross section for the 803.1-keV transition in ${ }^{206} \mathrm{~Pb}$. Right: a zoom on the region up to $1.1 \mathrm{MeV}$. Pictures taken from Ref. [13].

Table 1. Measurements performed at the GELINA neutron source using GAINS spectrometer. The status of the data is indicated and also some observations.

\begin{tabular}{c|c|c}
\hline Isotope & Reference & Observations \\
\hline${ }^{208} \mathrm{~Pb}$ & {$[12]$} & Comissioning of the digital acquisition \\
${ }^{206} \mathrm{~Pb}$ & {$[13]$} & \\
${ }^{209} \mathrm{Bi}$ & {$[14]$} & Investigation of level densities around $\mathrm{S}_{n}$ \\
${ }^{52} \mathrm{Cr}$ & {$[15]$} & Comparisons with charged-particles-induced reactions \\
${ }^{56} \mathrm{Fe}$ & {$[10]$} & Investigation of level densities around $\mathrm{S}_{n}$ \\
${ }^{57} \mathrm{Fe}[a]$ & & Dackground investigations for the $0 \nu \beta \beta$ experiments (see the text) \\
${ }^{28} \mathrm{Si}$ & {$[16]$} & Development of a standard $\gamma$-ray reference cross section \\
${ }^{24} \mathrm{Mg}$ & {$[17]$} & Development of a standard $\gamma$-ray reference cross section \\
${ }^{23} \mathrm{Na}$ & {$[18]$} & The highest $\gamma$ ray energy measured with GAINS $(6128 \mathrm{keV})$ \\
${ }^{76} \mathrm{Ge}$ & {$[19,20]$} & B \\
${ }^{12} \mathrm{C}^{[b]}$ & {$[21]$} & \\
${ }^{7} \mathrm{Li}$ & {$[22,23]$} &
\end{tabular}

more recently also with EMPIRE [25]. These reaction codes are general purpose codes that allows generating cross sections for a comprehensive set of reaction channels. The calculations of these reaction cross sections are based on a set of input parameters. In most of the cases it was not our intention to fine tune the parameters in order to obtain good fits but rather to test the performance of the default parameters and to report the result to the authors, especially when discrepancies occurred. Each measurement had some particularities. For example, in the case of ${ }^{206} \mathrm{~Pb}$, the presence of an isomeric level made impossible the measurements with the TOF technique. As a consequence, the contribution of this level to the total inelastic cross section will be missing and, as expected, TALYS indicated higher cross sections for certain transitions. After suppressing in TALYS the contribution of this level, the agreement was excellent (Fig. 3), giving confidence both for the measurement and for the assessment by TALYS for the missing level.

Among the measured targets, ${ }^{16} \mathrm{O}$ has the highest excited states ( $\gamma$ ray at $6128 \mathrm{keV}$ ). That required a careful calibration in efficiency at high energies and a larger range for amplitudes. An opposite situation exists in ${ }^{57} \mathrm{Fe}$ where the first excited state is at $14 \mathrm{keV}$. We could observe the line at $14 \mathrm{keV}$ with a source of ${ }^{57} \mathrm{Co}$ decaying to ${ }^{57} \mathrm{Fe}$ but, unfortunately, the background created by the beam on target was such that the level was completely unobservable during the data taking. Such and other similar situations determined us to investigate the possibility of building an ancillary detector for conversion electrons for which the work is in progress. In some cases, when we used natural targets (not separated isotopes), the (n, $2 \mathrm{n} \gamma$ ) reaction on a heavier isotope may overlap the results for a lighter one, e.g. ${ }^{56,57} \mathrm{Fe}$, ${ }^{n a t} \mathrm{Ti}$ target. However, this happens only at rather high energies due to the high $(\mathrm{n}, 2 \mathrm{n})$ threshold. It is interesting to remark that the measurements of inelastic neutron cross sections led also to additional results. Some of them are briefly mentioned below. The neutrinoless double $\beta$ decay expected from ${ }^{76} \mathrm{Ge}$ has Q-value of $2039.06 \mathrm{keV}$. All experiments take extreme precautions for all possible sources of background generated by the used on site materials. We scanned our measurements on ${ }^{206} \mathrm{~Pb}$, ${ }^{56} \mathrm{Fe},{ }^{28} \mathrm{Si},{ }^{24} \mathrm{Mg}$ and ${ }^{12} \mathrm{C}$ to see if neighboring lines do not appear in the spectra [20]. The results are shown in Fig. 4 and we hope that they bring clarifications concerning possible sources of background from the studied materials. In some light nuclei like ${ }^{24} \mathrm{Mg}$ or ${ }^{56} \mathrm{Fe}$, the level densities at low excitation energy are such that individual resonant scattering structures on various levels can be counted. We successfully did that and compared the obtained result with model predictions for level densities. For some reactions 


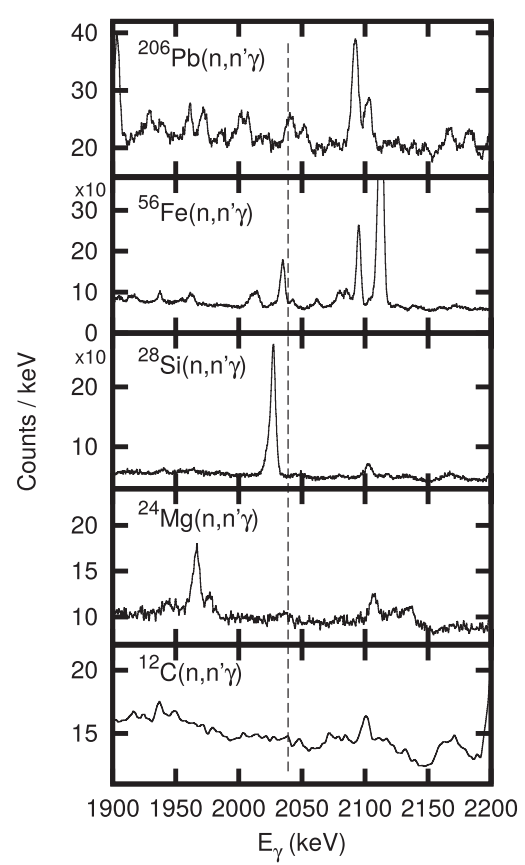

Figure 4. $\gamma$ spectra around the $\mathrm{Q}_{\beta \beta}\left({ }^{76} \mathrm{Ge}\right)=2039.06 \mathrm{keV}$ obtained in various neutron inelastic scattering experiments. Picture taken from Ref. [20].

we were interested to compare the (n, $\left.n^{\prime} \gamma\right)$ results with the ones from a reaction leading to the same compound nucleus but initiated by charged particles [16]. The studied reactions were:

- ${ }^{28} \mathrm{Si}\left(\mathrm{n}, \mathrm{n}^{\prime} \gamma\right)$ at GELINA;

- ${ }^{25} \mathrm{Mg}(\alpha, \mathrm{n} \gamma)$ at the 9MV IFIN-HH Tandem in Bucharest.

Although the energy domains are different due to the Coulomb barrier, in the overlap zone a comparison is still possible and we compared them also with model calculations. Last but not least, we performed a detailed study to determine the covariances. This study, though oriented rather to the covariances induced by our data taking and treatment than deterministic was a good and useful first exercise. The results were published as a JRC report.

\section{Conclusions and perspective}

This last decade was very fruitful for producing data for inelastic neutron cross sections. GAINS became a true and reliable data factory. Great improvements were progressively applied to the experimental setup and also to the DAQ and data treatment. A large variety of targets were measured and most of the results were published and uploaded in EXFOR. The results were compared with the predictions of TALYS leading to improvements of the code. This activity also includes $\mathrm{PhD}$ students and postdocs, having a pronounced educational/professional formation character. Researchers of different nationalities worked together and formed an ambitious and competitive team. There are also future plans: the mentioned detector for conversion electrons is a priority and will greatly enlarge the possibility to obtain information for nuclei with low lying, strongly converted transitions to the ground state or for E0 transitions. After moving GAINS to $100 \mathrm{~m}$ we hope to be able to measure coincidences, especially for the case of thick samples. Finally, the series of measurements will continue, a neutron inelastic cross section measurement on ${ }^{54} \mathrm{Fe}$ being ongoing.

C. Borcea acknowledges support by the Romanian Ministry of Education and Research through contract no. PN-II-ID-PCE2011-3-0370 (UEFISCDI).

\section{References}

[1] The Generation IV International Forum, online: http: //www.gen-4.org/

[2] Nuclear Energy Agency, OECD, Nuclear Data High Priority Request List, online: http: //www.nea.fr/dbdata/hprl/

[3] J.K. Tuli, Evaluated nuclear structure data file: A Manual for Preparation of Data Sets, BNL-NCS51655-01/02 Rev., Brookhaven National Laboratory (2001)

[4] A. Bensussan, J.M. Salome, Nucl. Instrum. Methods 155, 11 (1978)

[5] M. Flas̆ka, Ph.D. thesis, Slovak University of Technology in Bratislava, Published by IOS Press under the imprint Delft University Press, 2006

[6] D. Ene, C. Borcea, S. Kopecky, W. Mondelaers, A. Negret, A.J.M. Plompen, Nucl. Instrum. Methods Phys. Res. A 618, 54 (2010)

[7] A. Olacel, Ph.D. thesis, Faculty of Physics, University of Bucharest (2015)

[8] A.J.M. Plompen, N. Nankov, C. Rouki, M. Stanoiu, C. Borcea, D. Deleanu, A. Negret, P. Dessagne, M. Kerveno, G. Rudolf, J.C. Thiry, M. Mosconi, R. Nolte, J. Kor. Phys. Soc. 59, 1581 (2011)

[9] D. Deleanu, C. Borcea, Ph. Dessagne, M. Kerveno, A. Negret, A. J. M. Plompen, J.C. Thiry, Nucl. Instrum. Methods Phys. Res. 624, 130 (2010)

[10] A. Negret, C. Borcea, Ph. Dessagne, M. Kerveno, N. Nankov, A. Olacel, A.J.M. Plompen, C. Rouki, Nucl. Data Sheets 119, 179 (2014)

[11] L.C. Mihailescu, L. Olah, C. Borcea, A.J.M. PLompen, Nucl. Instrum. Methods Phys. Res. A 531, 375 (2004)

[12] L.C. Mihailescu, C. Borcea, P. Baumann, Ph. Dessagne, E. Jericha, H. Karam, M. Kerveno, A.J. Koning, N. Leveque, A. Pavlik, A.J.M. Plompen, C. Qutel, G. Rudolf, Nucl. Phys. A 811, 1 (2008)

[13] A. Negret, L.C. Mihailescu, C. Borcea, Ph. Dessagne, K.H. Guber, M. Kerveno, A.J. Koning, A. Olacel, A.J.M. Plompen, C. Rouki, G. Rudolf, Phys. Rev. C 91, 064618 (2015)

[14] L.C. Mihailescu, C. Borcea, A.J. Koning, A. Pavlik, A.J.M. Plompen, Nucl. Phys. A 799, 1 (2008)

[15] L.C. Mihailescu, C. Borcea, A.J. Koning, A.J.M. Plompen, Nucl. Phys. A 786, 1 (2007)

[16] A. Negret, C. Borcea, D. Bucurescu, D. Deleanu, Ph. Dessagne, D. Filipescu, D. Ghita, T. Glodariu, M. Kerveno, N. Marginean, R. Marginean, C. Mihai, S. Pascu, A.J.M. Plompen, T. Sava, L. Stroe, Phys. Rev. C 88, 034604 (2013)

[17] A. Olacel, C. Borcea, P. Dessagne, M. Kerveno, A. Negret, A.J.M. Plompen, Physical Review C 90, 034603 (2014) 
[18] C. Rouki, P. Archier, C. Borcea, C. De Saint Jean, J.C. Drohé, S. Kopecky, A. Moens, N. Nankov, A. Negret, G. Noguère, A.J.M. Plompen, M. Stanoiu, Nucl. Instrum. Phys. Res. A 672, 82 (2012)

[19] C. Rouki, A.R. Domula, J.C. Drohe, A.J. Koning, A.J.M. Plompen, K. Zuber, Phys. Rev. C 88, 054613 (2013)

[20] A. Negret, C. Borcea, A.J.M. Plompen, Phys. Rev. C 88, 027601 (2013)

[21] M. Nyman, F. Belloni, D. Ichinkhorloo, E. Pirovano, A.J.M. Plompen, C. Rouki, Phys. Rev. C 93, 024610 (2016)

[22] A. Olacel, F. Belloni, C. Borcea, A. Negret, N. Nyman, E. Pirovano, A.J.M. Plompen, JRC Technical report, JRC data for the Ti-48 standard,
Luxembourg: Publications Office of the European Union (2015)

[23] A. Olacel et al., in Proceedings of International Conference on Nuclear Data for Science and Technology (Bruges, Belgium, 2016), accepted to be publised in EPJ Web of Conferences

[24] A.J. Koning, S. Hilaire, M.C. Duijvestijn, International Conference on Nuclear Data for Science and Technology, April 22-27, 2007, Nice, France, editors O. Bersillon, F. Gunsing, E. Bauge, R. Jacqmin, S. Leray, EDP Sciences, p. 211-214 (2007)

[25] M. Herman, R. Capote, B.V. Carlson, P. Obložinsk'y, M. Sin, A. Trkov, H. Wienke, V. Zerkin, Nucl. Data Sheets 108, 2655 (2007) 